\title{
Editorial
}

\section{Macroscopic/Mesoscopic Computational Materials Science Modeling and Engineering}

\author{
Jian-Ming Lu, ${ }^{1}$ Jyh-Ming Ting, ${ }^{2} \mathrm{Mo} \mathrm{Li}^{3}{ }^{3}$ and Eugene Kogan ${ }^{4}$ \\ ${ }^{1}$ National Center for High-Performance Computing, Tainan 74147, Taiwan \\ ${ }^{2}$ Department of Materials Science and Engineering, National Cheng Kung University, Tainan 70101, Taiwan \\ ${ }^{3}$ School of Materials Science and Engineering, Georgia Institute of Technology, Atlanta, GA 30332-0245, USA \\ ${ }^{4}$ Department of Physics, Bar-Ilan University, Ramat-Gan 52900, Israel
}

Correspondence should be addressed to Jian-Ming Lu; 0403817@narlabs.org.tw

Received 1 November 2015; Accepted 17 November 2015

Copyright (C) 2015 Jian-Ming Lu et al. This is an open access article distributed under the Creative Commons Attribution License, which permits unrestricted use, distribution, and reproduction in any medium, provided the original work is properly cited.

The goal of this special issue is to provide the platform for international discussion and exchange of information/ experience on the simulation of phenomena in and characteristics of materials at macroscopic and mesoscopic scales. In particular, these publications are the exhibitions and wide materials science and engineering of innovative, improved, and elegant research in macroscopic and/or mesoscopic computational materials science and engineering, containing the most advanced mathematical modeling methods and numerical methodology developments.

For the sake of investigation of phenomena in materials science modeling and engineering at the macroscopic and mesoscopic scales, several powerful numerical tools, including first principle calculations, quantum molecular dynamics, molecular dynamics and molecular mechanics simulations, Monte Carlo method, finite element method, finite difference method, finite volume method, spectral element method, boundary element method, and computational fluid dynamics, are employed. Meanwhile, these tools are also applied on the research of materials (fluids, metals, polymers, rocks, ceramics, composites, biomaterials, nanomaterials, etc.), their structures (functional solids, soft matter, multiphase materials, tribology, coatings, etc.), and their characteristics (thermal, electronic, energetic, physical, structural, chemical, magnetic, or optical properties or a complicated combination of any of these properties).

The analytical and numerical analyses and results of functional elements of these materials constitute the main and outstanding highlights of this special issue; moreover, important computational studies of others aspects also are brilliant and impressive, such as imperfections in materials and their resulting limitations, novel processes for advanced materials synthesis, engineering of material properties, environmental consideration in material performance, and solid materials on sintering/melting process during three-dimensional printing. It enables interdisciplinary collaboration between science and engineering technologists in the academic and industrial fields.

\section{Acknowledgments}

We thank all authors for their new research work submitted to this special issue. And we are also very grateful to the reviewers for their professional comments for the manuscripts.

Jian-Ming Lu
Jyh-Ming Ting
Mo Li
Eugene Kogan




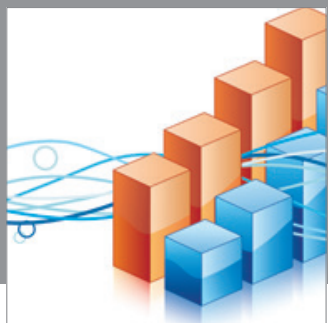

Advances in

Operations Research

mansans

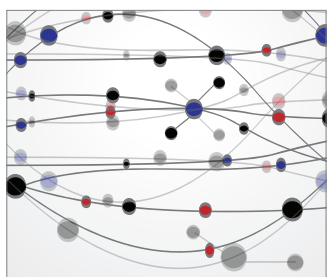

The Scientific World Journal
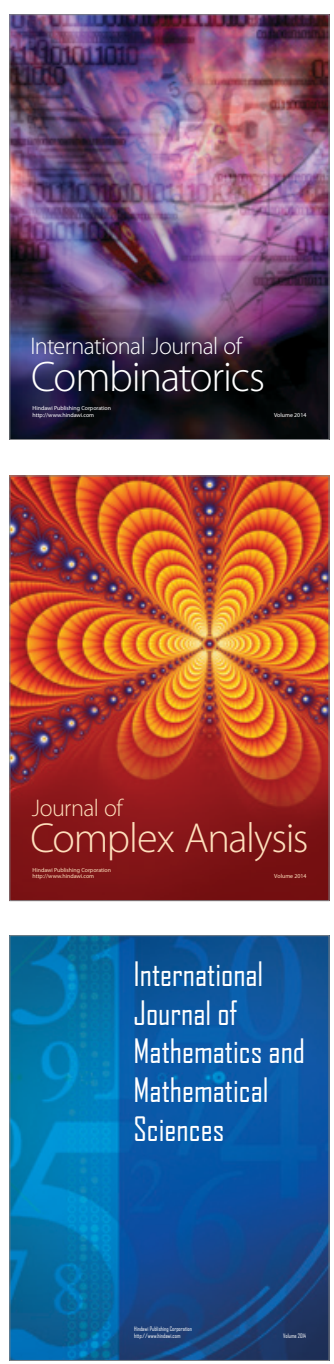
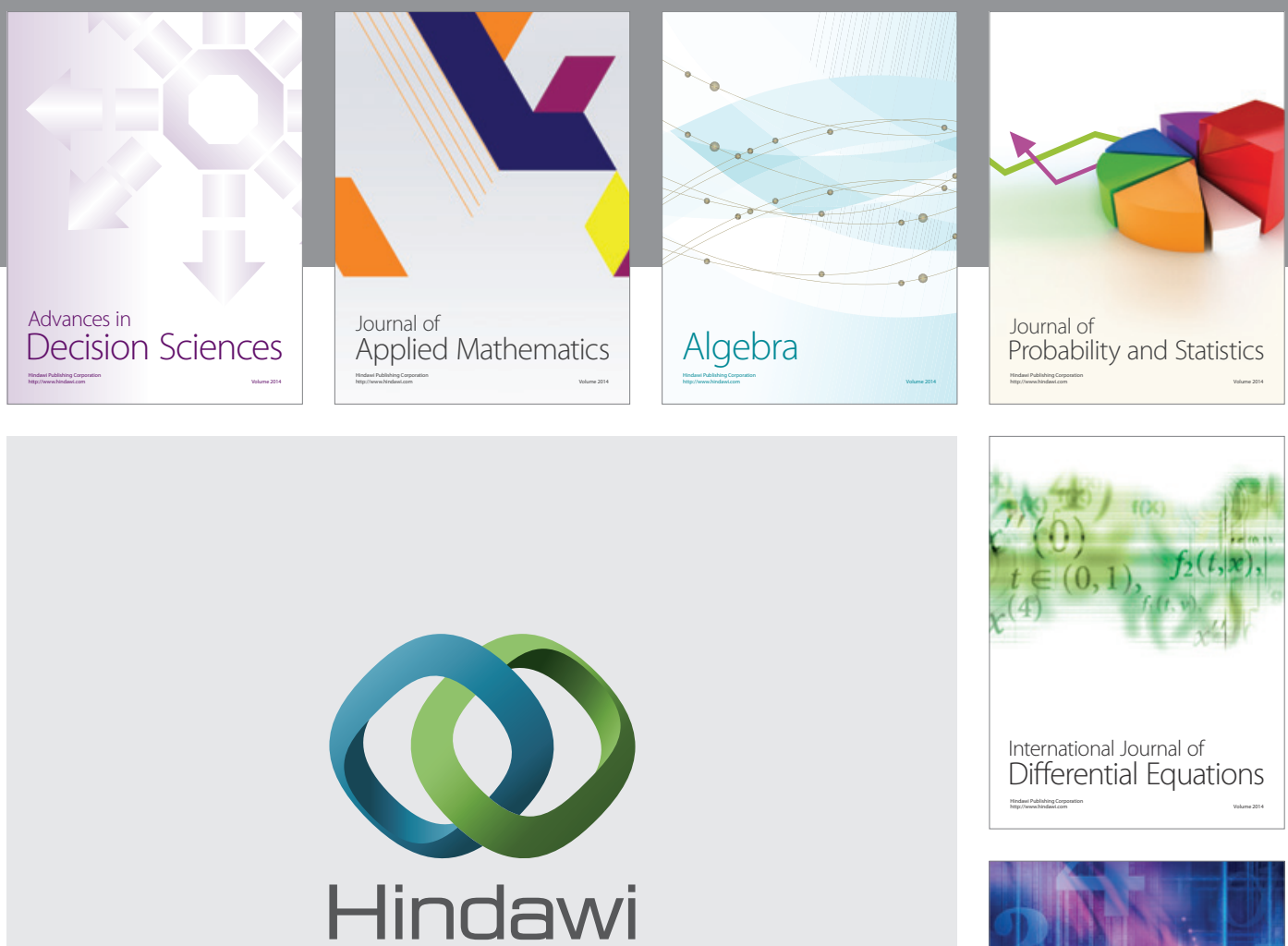

Submit your manuscripts at http://www.hindawi.com
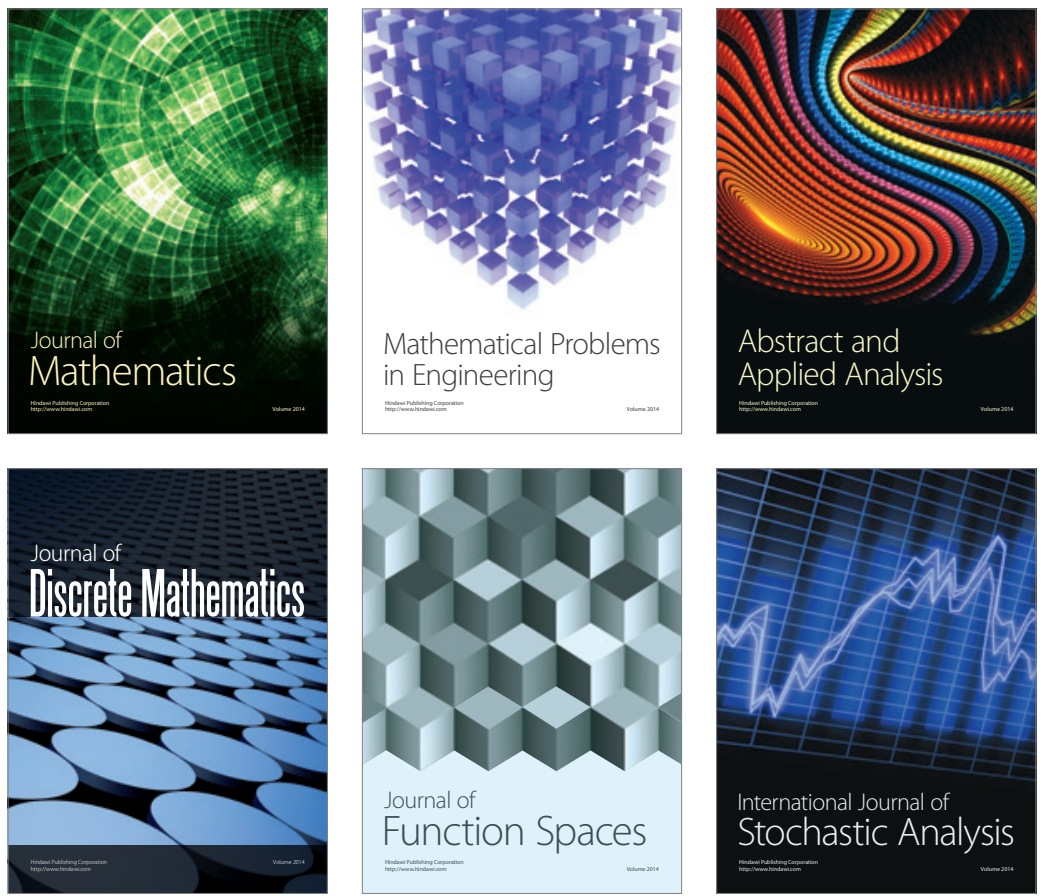

Journal of

Function Spaces

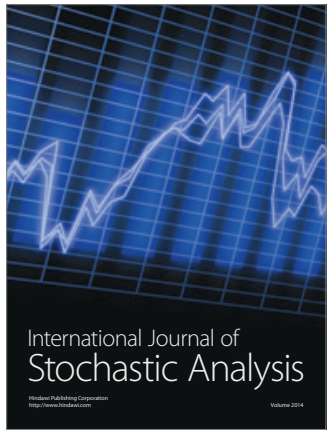

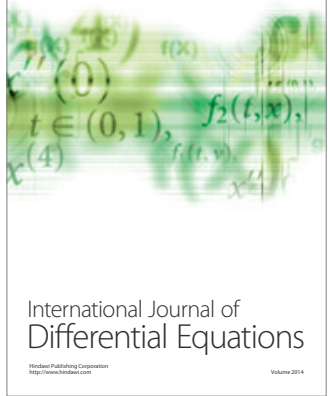
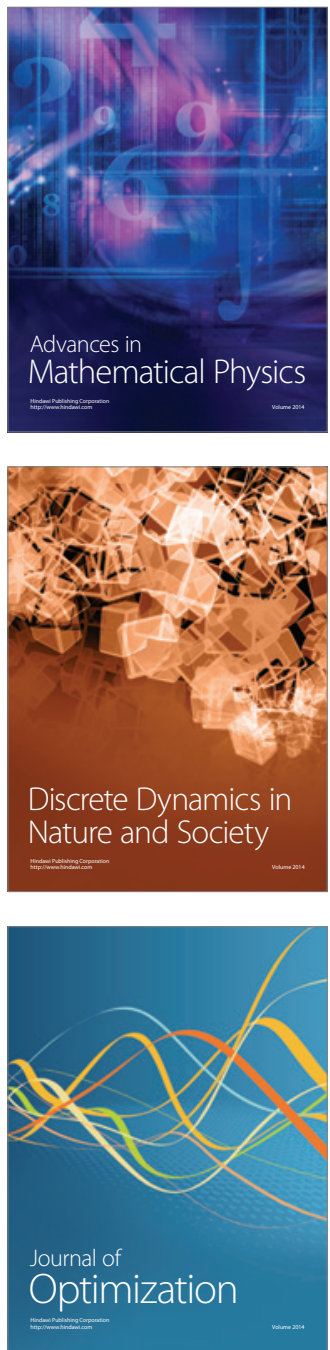